\title{
Impact of Extreme Electrical Fields on Charge Density Distributions in Alloys
}

\author{
Claudia Loyola ${ }^{1}$, Joaquín Peralta ${ }^{1}$, Scott R. Broderick ${ }^{2}$, and Krishna Rajan ${ }^{2}$ \\ 1. Departamento de Física, Facultad de Ciencias Exactas, Universidad Andrés Bello, Santiago, Chile. \\ 2. Dept. of Materials Designs and Innovation-University at Buffalo: State University of New York, USA.
}

The purpose of this work is to identify the field evaporation mechanism associated with charge density distribution under extreme electric fields, linking atom probe tomography (APT) experiments with density functional theory (DFT) computational modeling. We show how the evaporation field of atoms is highly related to the charge density by comparing the directionality and localization of the electrons with the evaporation of single ions versus dimers. The mechanism is an important component of the reconstruction process of APT data, which is partially dependent on the input evaporation fields of the atoms involved in the experimental procedure. Particularly we will study the case of $\mathrm{L}_{2}-\mathrm{Al}_{3} \mathrm{Sc}$ surface with different ad-atom configurations on the surface. We show that $\mathrm{Al}-\mathrm{Al}$ atom pairs on the surface are more likely to evaporate as dimers than Al-Sc ad-atoms. We show that this is due to Al-Al having a shared charge density, while Al-Sc has an increased density localized around the Sc atoms, with both effects provided by the intensity of the electric field applied on the surface.

Previously we used atomistic modeling to discriminate the evaporation field for single ion evaporations versus dimer evaporations in APT [1], thereby providing a more accurate input into the data reconstruction. This work builds on that prior work by now defining the mechanism which leads to different relative bonds strengths and different evaporation pathways under extreme electric field environments. By performing DFT calculations and calculating the change in charge density at the surface as a function of changing electric field, we are able to correlate the bonding properties under extreme environments with the field evaporation physics. The computations were made by the use of Quantum-ESPRESSO [2] for DFT with the generalized gradient approximation (GGA). The structure cell contained 80 atoms in the $\langle 111\rangle$ direction oriented along the $\mathrm{z}$-axis, with a vacuum in that direction of $15 \mathrm{~A}$, as shown Figure 1a. Four different configurations of ad-atoms on the surface have been considered in our study: Al, Sc, Al-Al, and Al-Sc. The chosen cutoff energy for the simulations was 200 Rydberg and the Marzari-Vanderbilt scheme was employed [3]. Dipole correction was used to incorporate the electric field in the simulations [4].

To observe the effects of high electric field on the charge distribution on the ad-atom configurations and the surface, different values of electric fields were considered based on the previous simulations [1], which corresponds to: Al - $29 \mathrm{~V} / \mathrm{nm}, \mathrm{Sc}-32 \mathrm{~V} / \mathrm{nm}, \mathrm{Al}-\mathrm{Al}-25 \mathrm{~V} / \mathrm{nm}$, and Al-Sc $-36 \mathrm{~V} / \mathrm{nm}$. The electronic charge density and the electronic localization function (ELF) were calculated in order to characterize the bond breaking process between the evaporating ions and the $\mathrm{Al}_{3} \mathrm{Sc}$ surface when a high electric field is present. To determine the charge density of the bond between the dimer and the surface, three different charge densities have been calculated. The first is an initial charge density $\rho_{0}$ that corresponds to the charge density of the full system, the second is the charge density associated to the surface without the dimer $\rho_{1}$, and third is the charge density of only the dimer $\rho_{2}$. The final charge density of the bond was determined using $\rho_{\text {bond }}=\rho_{0}-\rho_{1}-\rho_{2}$. To study the effects of high electric fields, we compare our results with experimental data provided by Atom Probe Tomography (APT). To correlate the DFT charge density calculations with the APT experimental data, we compare the ion evaporation map with the DFT results, as shown Figure 1b. A direct comparison between Al-Al dimers 
and Al-Sc dimers can be made by comparing $\mathrm{Al}^{2+}$ and $\mathrm{Al}^{+}$with $\mathrm{Sc}^{2+}$ and $\mathrm{Al}^{+}$. The reason is that the number of $\mathrm{Al}^{2+}$ hits and $\mathrm{Sc}^{2+}$ hits in the experiment were nearly equivalent, as shown Figure $1 \mathrm{~b}$. Therefore, any change in dimer concentration for these two points is due to increased number of dimers, and not a result of increased atomic concentration in the material.

The results provide the change in bonding and the mechanism for bond breaking under extreme electric field. We have also studied the charge distribution of bonds between ad-atoms and the surface to identify the evaporation pathways. Our results indicate that for $\mathrm{Al}_{3} \mathrm{Sc}$, the $\mathrm{Al}$ - $\mathrm{Al}$ dimer evaporation occurs due to a strengthening of the bond between the $\mathrm{Al}$ atoms in the presence of an electric field, while the Al-Sc dimer evaporation is due largely to a weakening of neighboring Al-Al bonds. However, the charge localization around the Sc atom results in primarily single ion evaporations for the Al-Sc case. These results provide the bonding mechanism corresponding to the calculated electric fields of single ion evaporations versus dimer evaporations which improve the APT reconstruction, while also providing guidelines for surface chemistry design for materials used in extreme field applications. [5]

References:

[1] J Peralta, S.R. Broderick, K. Rajan, Ultramicroscopy 132 (2013) p. 143.

[2] P. Gianozzi, S. Baroni, N. Bonini, et al, Journal of Physics: Condensed Matter 29 (1009) p. 395502.

[3] N. Marzari, D. Vanderbilt, M. Payne, Physical Review Letters 79 (1997) 1337-1340.

[4] L. Bengsston, Physical Review B 59 (1999) 12301-12304

[5] TC \& JP acknowledge funding from Proyecto Fondecyt Iniciacion 11130501.

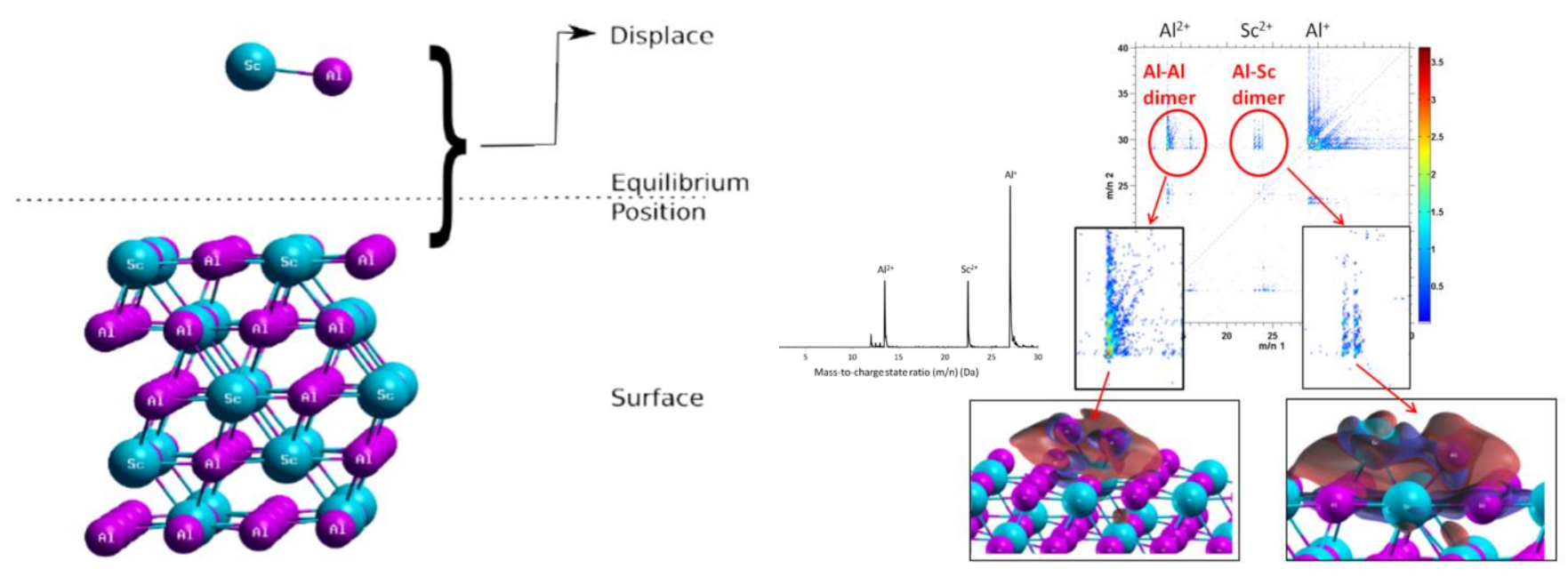

(a)

(b)

Figure 1. (a) Initial slab of $\mathrm{Al}_{3} \mathrm{Sc}$ with $\mathrm{A}-\mathrm{B}-\mathrm{C}$ layer scheme for the surface. The evaporated ion or dimer is placed at the surface and an electric field is applied. (b) Mass-to-charge spectra of the sample from APT, and the integration of APT experimental measurements with DFT data. The inset regions focus on the Al-Al dimer evaporations compared to Al-Sc dimers, with the overall chemistry of the material for these two regions being nearly equivalent. The number of Al-Al dimers is seen to be significantly higher. The DFT results describe the reason for this being the charge localization (shown as dark blue) around the Sc atom, resulting in Sc evaporating as a single ion. This figure demonstrates how DFT provides a description of evaporation mechanisms, which can then be applied to interpretation of the experimental data. 